\title{
Primary Histoplasmosis of Vocal Cord in an Immunocompetent Elderly Man- A case Report with Literature Review
}

\author{
SHAH KHALED HABIB, ${ }^{1}$ SHAMSUL AREFIN PATWARY, ${ }^{2}$ MOHAMMAD ASHIK IMRAN KHAN, ${ }^{1}$ MD. TITU MIAH ${ }^{3}$ RATAN \\ DAS GUPTA, ${ }^{3}$ HAM NAZMUL AHASAN ${ }^{4}$
}

\begin{abstract}
:
Histoplasmosis is a fungal infection primarily acquired through inhalation, and is rarely encountered outside endemic areas. In non-endemic areas, histoplasmosis poses a diagnostic challenge, especially since the presenting lesions can mimic carcinoma and other chronic infections including tuberculosis. We report an unusual case of isolated vocal cord histoplasmosis in a 60 year old immunocompetent man from Bangladesh presenting with hoarseness of voice.
\end{abstract}

Keyword: Histoplasmosis, larynx/vocal cord, immunocompetent, Bangladesh

\section{Introduction:}

Granulomatous diseases caused by infectious agents are being seen more frequently encountered than before. Infection by histoplasma capsulatum is one such granulomatous disease. ${ }^{1}$ The infection is acquired by inhalation of dust particles from soil contaminated with bird or bat droppings that contain small spores (microconidia), the infectious form of fungus. ${ }^{2,3}$ According to Goodwin Jr. et al, it was Monbreun, in 1934, who first saw that the histoplasma had two ways of growing, in room temperature (as a filamentous fungus) and in body temperature (like yeasts). ${ }^{4}$ The fungus invades the reticular-endothelial system.

Histoplasma is known to occur naturally in caves inhabited by bats. The outbreaks of histoplasmosis have been reported in cave explorers. ${ }^{5}$ Majority of primary infections in immunocompetent hosts are asymptomatic or may present with flu like illness. ${ }^{5}$ Hematogenous (disseminated) histoplasmosis occurs in immunocompromised individuals. ${ }^{5,6,7}$ Patients with AIDS are susceptible to disseminated infection with histoplasma, which is a major opportunistic pathogen in this disease. ${ }^{2}$ Laryngeal involvement is usually associated with dissemination. ${ }^{1,5,8}$ The primary histoplasmosis of larynx is an extremely rare condition. $3,7,8$ Histoplasma infection is even rarer in Bangladesh where only four cases have been reported so far and, to best of our knowledge, this is the first case report from Bangladesh on vocal cord Histoplasmosis. ${ }^{9,10,11,12}$ Here we report a case of 60 years old immunocompetent man with a background of tuberculosis who received 2 episodes of anti TB medications presenting with hoarseness of voice leading to the diagnosis of primary histoplasmosis of the vocal cord from biopsy.

\section{Case Report:}

A 60 year old man got admitted with the complaint of progressive hoarseness of voice for the 6 months. He had a history of Tuberculosis for which he had been treated with completed course of anti TB drugs in two occasions about 20 years and 6 months back respectively. Grounds on which tuberculosis diagnosis was made was not clear as previous medical records were not available. During the last six months he had no fever, night sweats, weight loss or any other systemic symptoms and signs suggestive of tuberculosis. His general examination was normal. His indirect laryngoscopy revealed ulcerative growth in the left vocal cord. Both vocal cords were mobile. Piriform fossa, aryepiglottic fold and vestibular fold were normal. Then a direct laryngoscopy was done which showed ulcerative growth of the left vocal cord and a small biopsy specimen was taken and subjected to histopathological examination. Microscopic examination revealed two pieces of vocal cord tissue lined by squamous epithelium. The stroma revealed epitheliod granuloma admixed with chronic inflammatory cells including many eosinophils. There were tiny round to oval bodies intracellularly as well as extracellularly seen on PAS and Gomori methanamine silver stains morphologically consistent with histoplasmosis (Fig.-1). His chest x ray revealed diffuse patchy opacities seen all over the lung fields

1. Post-graduate trainee, Department of Medicine, Dhaka Medical College

2. FCPS (Medicine) course, Dhaka Medical College.

3. Assistant Professor, Department of Medicine, Dhaka Medical College.

4. Professor, Department of Medicine, Dhaka Medical College.

Correspondence: Mohammad Ashik Imran Khan, post graduate trainee, DMCH; E mail- ashikmrn@yahoo.com 


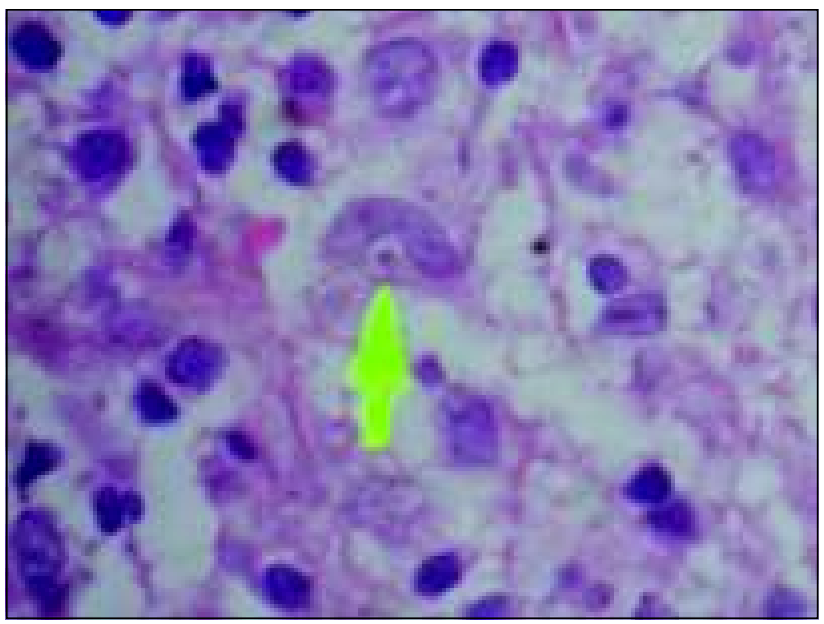

Fig.-1 : Showing histoplama

(Fig.-2a). This led to the suspicion of disseminated histoplasmosis and warranted some more investigations. His chest CT scan revealed fibrosis, bulla and fibrotic nodules in both lungs. It also showed subtle bronchiectatic changes in posterior segment of right upper lobe and focal emphysematous changes in both lungs remarking as a sequelae of pulmonary tuberculosis (Fig.-2b). His bronchoscopy showed normal tracheobronchial tree other than a white patch over the left vocal cord. Microscopy study of BAL showed lymphocytes, few neutrophils and granular proteinacious material in background. Culture of BAL yielded pseudomonas which was sensitive to amikacin,

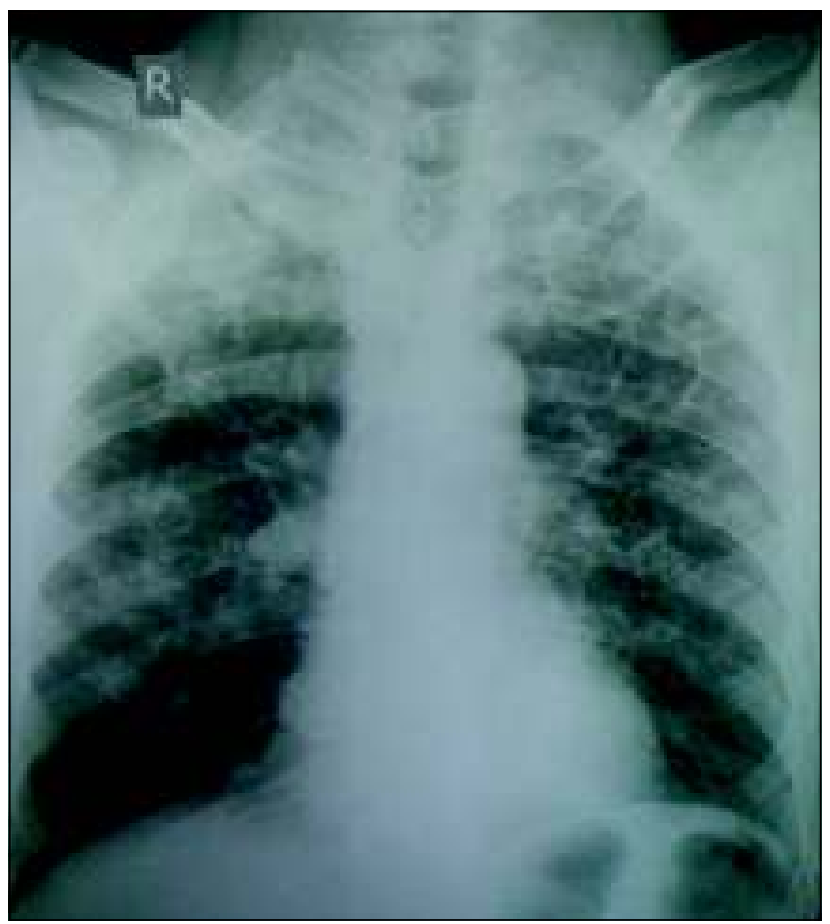

Fig.-2a : Showing B/L diffuse patchy opacity

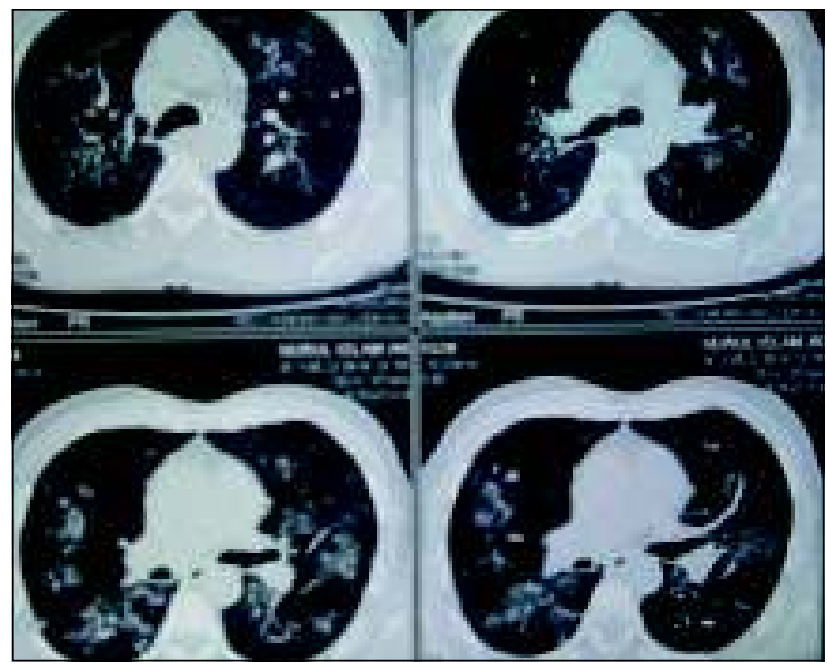

Fig.-2b: Showing sequelae PTB

levofloxacin and gentamycin. Gram, AFB and fungal stains were all negative. Disseminated histoplasmosis and an immunocompromised state were excluded by conducting a complete blood count, blood sugar, liver function tests, chest X-ray and enzyme-linked immunosorbant assay (ELISA) for HIV, which were all normal. The final diagnosis was primary vocal cord histoplasmosis with pseudomonas respiratory infection. The patient had then received levofloxacin $500 \mathrm{mg}$ PO twice daily for 10 days. For his histoplasmosis he received 14 doses of $0.5 \mathrm{mg} / \mathrm{kg}$ Amphotericin B intravenously every alternate day. The patient's symptoms gradually improved after the treatment and he was discharged on itraconazole $200 \mathrm{mg}$ PO twice daily for 12 weeks and advised for subsequent follow up visits.

\section{Discussion:}

Histoplasmosis is a common granulomatous disease of worldwide distribution caused by a dimorphic fungus Histoplasma capsulatum. ${ }^{1}$ Darling first discovered the organism in 1905 and thought causative agent was protozoan, later it proved to be fungus. ${ }^{13} \mathrm{He}$ first named and described the organism in 1906 from postmortem studies of liver, spleen, lungs, lymph nodes and adrenal glands. The first case of histoplasmosis was reported in 1906 in United States. ${ }^{8}$ In 1950 's although the fungal nature of the disease was well established but it was considered as a deficiency disease and treated with vitamins and mineral supplements as no specific treatment was available in that era. ${ }^{13}$ In the present era with availability of modern antifungal agents the condition is completely curable. The pathogenesis of histoplasmosis is incompletely understood. It is known that macrophages are the major targets of histoplasma capsulatum. The fungus expresses heat shock protein 60 (hsp60) on its cell surface that binds to alpha 2 integrins 
on the surface of macrophages. Histoplasma induces macrophages to secrete TNF which stimulates and recruits other macrophages to kill the histoplasma. ${ }^{2}$

After organism is inhaled there is a primary infection. There are two types of primary infection according to the number of organisms inhaled: Infection with a moderate number of organisms, and infection with a massive number of organisms inhaled, usually in bat caves or in hen houses. In the former, there is asymptomatic or mild respiratory infection with one or few primary pulmonary sites. In the latter, the picture is much worse, there are multiple and simultaneous primary sites, if the disease is not treated then, it progresses to cavitations or some type of spread disease. ${ }^{2}$ Interestingly our patient had no travel history to histoplasmosis endemic zone or intake of immunosuppressive drugs. His job was not consistent with exposure to fungal infections.

With the primary infection there is a fast and transitory systemic spread. For most patients the disease is limited to this point. For most immunocompetent patients, it is asymptomatic or is similar to a cold in its symptoms. ${ }^{14}$

In acute spread cases, usually in children below one year of age or in severely immunosuppressed patients, there is fever, weight loss, liver and spleen enlargement and pancytopenia, and the patient may develop shock, respiratory failure and disseminated intra-vascular coagulation (DIVC). In the cases when the infection extends - the acute spread, there may also be adrenalitis, endocarditis, meningitis, and ulcerations in the oral mucosa and in the intestine. In the chronic forms there is fever, weight loss, asthenia, liver and spleen enlargement and oral mucosa lesions are very common. In the acute forms, ulcerations were much less frequent. ${ }^{15}$

Chronic pulmonary histoplasmosis is very much similar to tuberculosis, with slow progressive fever, weight loss and respiratory symptoms with chest $\mathrm{x}$-rays showing areas of excessive cavitations and fibrosis. ${ }^{15}$ It must especially be considered in patients who do not respond to tuberculosis treatment, in whom the tuberculosis agent was not identified. ${ }^{16}$ Differently from the patients with the spread form of the disease, these here do not have the involvement of other organs (muco-cutaneous, meningitis, pericarditis, adrenal failure forms of the disease). Staloff et al. commented that the spread is very rare, as a clinically apparent infection, happening more frequently in immunosuppressed and elderly patients, and more commonly in men. When the infection spreads, the histoplasma can invade almost any organ. The areas commonly involved are: bone marrow, lymph nodes, adrenal glands and gastrointestinal tract, just as the tongue and oral mucosa. ${ }^{17}$ Although the initial chest X-ray of our patient raised suspicion of disseminated histoplasmosis further investigations including chest CT, Bronchoscopy and BAL study helped to rule out other possibilities.

Thus, laryngeal/vocal cord involvement occurs in the mucocutaneous form of the chronic spread disease. Common initial manifestations are: pain to swallow, hoarseness, gingival ulceration and dysphagia. ${ }^{18}$ It is also common for these patients to complain of weight loss, malaise and fatigue. ${ }^{19}$ Hepatoesplenomegaly can occur in $30-50 \%$ of the cases, as well as skin ulcerations. Mucosa ulcerations are the most frequent findings in these patients. They may last for long periods of time in any area of the larynx. Firm, painful ulcers, with elevated borders, involving the tongue, oral mucosa and the larynx are characteristic. Submucosa masses, with or without ulcerations may also appear - mimicking carcinomas. These ulcerations can involve the oral mucosa, the gum, the tongue, lips and pharynx. ${ }^{20}$ Though not very common, local involvement of laryngeal mucosa can represent the only sign of spread infection. During examination, the laryngeal mucosa can be pearl-white and edematous, or inflamed and ulcerated. The lesions can be similar to carcinoma or tuberculosis. Although most authors agree that the laryngeal manifestations indicate spread lesions, there are cases reported in which the laryngeal lesions were considered the primary ones. ${ }^{21-22}$ For Smith, Utz only $15 \%$ of the patients had isolated laryngeal lesions, however $42 \%$ manifested the oro-laryngeal disease. According to Stallof et al, Hutchinson (1952) described one case in which the disease happened specifically on the right vocal fold. ${ }^{23,24}$ Wihters, Pappas described a similar case, but one that did not have signs of spread. Bhalla et al., studying the causes of acquired non-neoplastic laryngotracheal stenosis noticed that histoplasmosis was also part of the differential diagnosis, together with sarcoidosis, tuberculosis, osteoplastic tracheoplasty and Wegener's granuloma. ${ }^{25,} 26$ In our patient only the vocal cords were involved. He had recurrent episodes of pulmonary tuberculosis but were successfully treated. Unfortunately the basis of the diagnosis on those occasions could not be evaluated due to lack of medical records.

The tuberculate microconidia are chraracteristic of $\mathrm{H}$. capsulatum but identification should be verified with the use of DNA probe which is a confirmatory test with high specificity. Measurements of antibodies against $\mathrm{H}$. capsulatum are accomplished by complement fixation and immunodiffusion. ${ }^{27}$ The only diagnostic certainty is achieved by isolating the microorganism. ${ }^{17}$ Biopsy shows granulomatous tissue, frequently necrosed, with an infiltrate of gigantic cells, lymphocytes, plasmocytic cells and a large number of macrophages. The organisms are not easily seen with the hematoxyline-eosin dye. However, with the Gomori 
silver methenamine, the macrophage appears with a variable number of oval and round bodies, surrounded by a clear zone. These bodies represent an intracellular hypha of the H. capsulatum. ${ }^{20}$ As all of these modalities are not possible in the context of diagnostic facilities available in Bangladesh, typical histopathological picture pointed towards the diagnosis in our patient. A clinical impression of tuberculosis/malignancy was made on the clinical grounds but other diagnostic tests ruled out theses possibilities and microscopic examination of Hematoxyline \& Eosin, Periodic Acid Schiff and silver stained sections revealed features consistent with histoplasmosis.

Laryngeal histoplasmosis must be treated similarly to the other forms of the disease. Although benign, there can be dissemination with severe or even fatal manifestations. Treatment is carried out with intravenousnous amphotericin B, $0.3-0.6 \mathrm{mg} / \mathrm{kg}$ of body weight per day, with a total dose of 2-4 mg. Mucosal lesions respond quickly (6-8 weeks), although there may be recurrences. ${ }^{20}$ Goodwin Jr. et al. reported that a serum level of amphotericin B of $1.56 \mathrm{mg} / \mathrm{mL}$ for 10 weeks, with a total of $1 \mathrm{~g}$ of intravenous medication, is usually enough for cure, or, when the serum level cannot be monitored, 50mg per day or three times a week can be an alternate regimen, at a total dose of $2.0 \mathrm{~g}$. Of their 84 cases treated with amphotericin B, 11 did not cure completely and required a new treatment. ${ }^{4}$ Review of literature revealed various regimens of treatments for laryngeal histoplamosis. Fletcher, Prussin suggested that in immunocompetent patients with laryngeal histoplasmosis, one or two months of Ketoconazole could be administered, and if there is improvement, treatment can remain for six months. ${ }^{16}$ Staloff et al. reported a case of histoplasmosis affecting the larynx only, treated with $200 \mathrm{mg}$ of Ketoconazole tid for 3 months good results. ${ }^{17}$ Negroni et al. treated 17 histoplasmosis patients with itraconazole, $100 \mathrm{mg}$ daily until clinical cure and, then, changed it to $50 \mathrm{mg} /$ day for six more months. All the infections were clinically cured, or improved greatly. 12 cases had clinical cure, 4 extreme reliefs and 1 patient, who interrupted treatment after 2 months of itraconazole, died. ${ }^{28}$

Very few cases of isolated/primary laryngeal histoplamosis without any pulmonary involvement have been reported till date. ${ }^{27}$ In our case also there was no pulmonary or systemic involvement. Patient responded well to systemic amphotericin B with complete remission of symptoms followed by oral itraconazole. Though, our experience with histoplasmosis treatment is limited but our patient tolerated the treatment well.

\section{Conclusion:}

Isolated laryngeal histoplasmosis is a rare entity. Because of its clinical simulation with malignancy it needs to be included in the differential diagnosis of neoplasms and chronic ulcers like mid-line granuloma, mucormycosis, lymphoma, syphilis, tuberculosis and other granulomatous diseases of upper aero digestive tract. A high index of suspicion of histoplasmosis should be kept in mind in cases of ulcerative lesions of the upper aero digestive tracts, where histological examination of the biopsies fails to show dysplasia or where there is a failure to respond to anti-tuberculosis treatment. Concerted effort is needed to setup diagnostic utilities for the diagnosis of the lurking danger of Histoplasmosis.

\section{Conflict of Interest: None.}

\section{References:}

1. Donegan JO, Wood MD. Histoplasmosis of larynx. Laryngoscope 1984;94(2pt1):206-09.

2. Kumar V, Husain AN. The lung: In; Kumar V, Abbas AK,Fausto N (eds.) Robbins and Cotran Pathological Basis of Disease 7th ed. WB Saunders Company Philadelphia, 2004;.pp. 711-72.

3. O'Hara CD, Allegretto MW, Taylor GD, Isotalo PA.Epiglotic histoplasmosis presenting in a non-endemic region: a clinical mimic of laryngeal carcinoma. Arch Pathol LabMed 2004;128(5):574-77

4. Goodwin Jr. RA, Shapiro JH, Thurman GH, Thurman SS, Prez RM. Disseminated Histoplasmosis: Clinical and pathologic correlations. Medicine [Baltimore] 1990; 59:133.

5. Gugnani HC. Histoplasmosis in Africa: A review. Indian J Chest Dis allied Sci 2001;42(4):271-74.

6. Wolf J, Blumberg HM, Leonard MK. Laryngeal histoplasmosis. Am J Med Sci 2004;327(3):160-62.

7. Hughes WT. Hematogenous Histoplasmosis in an immunocompromised child. J Pediatr 1984;105(4):569-75.

8. Withers BT, Pappas JJ, Erickson EE. Histoplasmosis primary in the larynx. Arch otorhinolaryngol $1963 ; 77: 25$ 28.

9. Subramaniam S, Abdullah AH, Hairuzah I. Histoplasmosis of larynx. Med J Malaysia 2005;60(3):386-88.

9. Md. Shahriar Mahbub, HAM Nazmul Ahasan, Md Titu Miah, Md Billal Alam, Ratan Das Gupta, Khan Mohammad Arif, Muntasir Hasnain. Disseminated Histoplasmosis. J Medicine 2010; 11: 70-73

10. Subramanian S, Abraham OC, Rupali P, et al. Disseminated Histoplasmosis. J Assoc Physicians India 2005;53:185-189

11. Rappo U, Beitler UR, Faulhaber JR, et al. Expanding the horizons of histoplasmosis: disseminated histoplasmosis in a renal transplant patient after a trip to Bangladesh. Transpl Infect Dis 2009;:1-6. 
12. Islam N, Chowdhury NA. Histoplasmosis from Bangladesh: a case report. Bangladesh Med Res Counc Bull 1982; 8: 2124

13. Roberts SE, Forman FS. Histoplasmosis a deficiency disease.Ann Otol Rhinol Laryngol 50;59(3):809-822.

14. Wolf J, Blumberg HM, Leonard MK. Laryngeal histoplasmosis. Am J Med Sci 2004;327(3):160-2.

15. Goodwin Jr. RA, Shapiro JH, Thurman GH, Thurman SS, Prez RM. Disseminated Histoplasmosis: Clinical and pathologic correlations. Medicine [Baltimore] 1990;59:133.

16. Fletcher SM, Prussin AJ. Histoplasmosis of the larynx treated with ketoconazole: a case report. Otolaryngol Head Neck Surg 1990;103:813-6.

17. Staloff RT, Wilborn A, Prestipino A, Hawkshaw M, Reinhardt JH, Cohn J. Histoplasmosis of the larynx. Am J Otolaryngol 1993;14:199-205.

18. Reibel JF, Jahrsdoerfer RA, Johns MM, Cantrell RW. Histoplasmosis of the larynx. Otolaryngol Head Neck Surg 1982;90 740-3.

19. Rajah V. Histoplasmosis of the oral cavity, oropharynx and larynx. The J Laryngol and Otol 1993; 107:58-61

20. Donegan JO, Wood MD. Histoplasmosis of the larynx. Laryngoscope 1984; 206-9.
21. Fletcher SM, Prussin AJ. Histoplasmosis of the larynx treated with ketoconazole: a case report. Otolaryngol Head Neck Surg 1990; 103:813-6.

22. Reddy P, Sutaria MK, Christianson CS, Brasher CA. Ann Otol Rhinol Laryngol 1970; 79:389-97.

23. Staloff RT, Wilborn A, Prestipino A, Hawkshaw M, Reinhardt JH, Cohn J. Histoplasmosis of the larynx. Am J Otolaryngol 1993;14:199-205.

24. Smith JW, Utz JP. Progressive disseminated histoplasmosis. A prospective study of 26 patients. Ann Intern Med 1972;76:557-65.

25. Withers BT, Pappas JJ, Erickson EE. Histoplasmosis primary in the larynx. Report of a case. Arch Otolaryngol 1963;77:25-8.

26. Bhalla M, Grillo HC, McLoud TC, Shepard JO, Weber AL, Mark EJ. Idiopathic laringotracheal stenosis: radiologic findings. AJR Am J Roentgenol. 1993;161:515-7.

27. Kauffman CA. Endemic mycoses after hemapoietic stem cell or solid organ transplantation. In: Bowden RA, Ljungman P, Paya CV, editors. Transplant infections. $2^{\text {nd }}$ edition. Philadelphia: Lippingcott Williams \& Wilkins; 2003. P.52434

28. Negroni R, Palmieri O, Koren F, Tiraboschi IN, Galimberti RL. Oral treatment of paracoccidioidomycosis and histoplasmosis with itraconazole in humans. Rev Infect Dis 1987;9 Suppl 1:S47-50. 